\title{
COMMENTARY
}

\section{Good primary mental health care is simple}

Is a GP whose reasoning is more complex better for patients with common mental disorders? The paper by Cape et a/ ${ }^{1}$ tries to shed some light on an area that is clinically highly relevant but that has received surprisingly little research attention. The key concept discussed by Cape et al is 'complexity'. They hypothesise that the 'more complex a GP's thinking about mental health problems is, the more likely it is that the GP will be able to help patients to make meaning of their problems in ways that may assist in easing their distress and helping them engage with potentially helpful treatments'. ${ }^{1}$ Their research idea is to link such increasingly complex thinking by the GP to increased clinical competence.

Although the idea of increasingly complex thinking is intuitively appealing and we would welcome new opportunities to help GPs to increase their clinical competence, we have severe doubts about the concept of 'complex thinking'.

Our first doubt concerns the rational focus ('thinking') of their hypothesis. Cape et al state that: 'A measure of GPs' thinking about mental health problems is needed." However, to interact meaningfully (and purposefully) with patients with common mental disorders, GPs need first to be able to manage feelings, and empathise. ${ }^{2}$ The key to better detection and management of mental health problems, for patients as well as for GPs, is to focus on feelings, rather than on thinking. Lack of competence is mostly due to a lack of empathy, and not due to complexity. The definition that Cape et al chose for complexity does not mention emotions or empathy at all. In many forms of psychotherapy, and especially in the most evidence-based forms such as cognitive behavioural therapy and interpersonal therapy, the therapist teaches the patient (in the here and now) how to control negative feelings. ${ }^{3}$ In simple but effective primary care treatment(s), such as problem-solving treatment, patients are encouraged to change their actual behaviour first, rather than their thinking. ${ }^{4}$ The patient experiences emotional control as a consequence, for instance, of a pleasurable activity. It may not be the complex thinking of the GP or the therapist that helps, but the patient's own actions. Patients learn to acknowledge symptoms, to define problems and goals, for instance to stop avoiding difficult issues at work, and to take steps towards achieving these goals. In the famous patientcentredness model, 'feeling' is also the first dimension to be addressed. ${ }^{5}$ Unfortunately, many GPs find this dimension the most difficult to discuss ('a conspiracy of silence'). ${ }^{6}$

Our second doubt concerns the 'black box' aspect of the concept of 'thinking': by its very nature, this concept is not countable. What exactly is complex thinking, and who defines it? For example, does it encompass the GP's competence to observe interpersonal cues or to empathise? A GP, a patient, or a third party may observe at least three different complexities in one single consultation. ${ }^{7}$

A third doubt is that many common mental disorders are self-limiting: although the GP (and the patient) might initially consider a mental health problem to be complex, a few weeks later many patients have solved their own problems. In that case, complex thinking by the GP was unnecessary.

This is an interesting area for research, but we contend that the opposite of complexity is required. Good collaborative primary mental health care is simple, and specific. Patients should be invited to: acknowledge their symptoms in the here and now, for instance by completing a simple and responsive questionnaire such as the Four Dimensional Symptom Questionnaire; ${ }^{8}$ discuss their specific ideas about the context of these symptoms; take specific steps to increase their motivation to control the symptoms; and monitor their symptoms closely. Good primary mental health care should focus more on symptoms, as in the management of other chronic health problems such as diabetes or hypertension, and not on more complexity.

\section{Harm van Marwijk,}

Associate Professor of General Practice, Department of General Practice, EMGO Institute, VU University Medical Centre,

Van der Boechorststraat 7, 1081 BT Amsterdam, the Netherlands.

\section{Berend Terluin,}

Senior Researcher, GP, Department of General Practice, EMGO Institute, VU University Medical Centre, Amsterdam.

\section{REFERENCES}

1. Cape J, Morris E, Burd M, Buszewicz M. Complexity of GPs' explanations about mental health problems: development, reliability, and validity of a measure. Br J Gen Pract 2008; 58: DOI: 10.3399/bjgp08X299281

2. Deveugele M, Derese A, De Bacquer D, et al. Is the communicative behavior of GPs during the consultation related to the diagnosis? A cross-sectional study in six European countries. Patient Educ Couns 2004; 54(3): 283-289.

3. Parker G, Fletcher K. Treating depression with the evidence-based psychotherapies: a critique of the evidence. Acta Psychiatr Scand 2007; 115(5): 352-359.

4. Mynors-Wallis LM, Gath DH, Day A, Baker F. Randomised controlled trial of problem solving treatment, antidepressant medication, and combined treatment for major depression in primary care. $B M J 2000 ; 320(7226): 26-30$.

5. Stewart M, Brown J, Weston W, et al. Patient-centered medicine, transforming the clinical method (2nd edn). Oxford: Radcliffe; 2003.

6. Demyttenaere K, Bruffaerts R, Posada-Villa J, et al. Prevalence, severity, and unmet need for treatment of mental disorders in the World Health Organization World Mental Health Surveys. JAMA 2004; 291(21): 2581-2590.

7. Van Marwijk H, Gercama A, Ader H, de Haan M. Mean clinical challenge rate and level of recognition of depression remain unchanged after two years of vocational training. Fam Pract 2001; 18(6): 590-591.

8. Terluin B, van Marwijk HW, Ader HJ, et al. The Four-Dimensional Symptom Questionnaire (4DSQ): a validation study of a multidimensional self-report questionnaire to assess distress, depression, anxiety and somatization. BMC Psychiatry 2006; 6: 34.

DOI: 10.3399/bjgp08X302691 